\title{
BLOCK STRUCTURE AND OBJECT ORIENTED LANGUAGES
}

\author{
Ole Lehrmann Madsen
}

Computer Science Department, Aarhus University, Aarhus, Denmark

\section{Introduction}

Simula 67 [Simula] and Smalltalk [Goldberg \& Robson 85] are two examples of programming languages that support the object-oriented programming style. The two languages are different in a number of ways: Simula contains Algol 60 as a subset and thus supports block structure, static (lexical) name binding, and compile-time type checking. Smalltalk has none of these features. Instead Smalltalk is more in the style of Lisp with a flat set of definitions (classes), dynamic name binding and run-time typechecking.

The purpose of this paper is to discuss the role of block structure in object oriented languages. In [Wulf \& Shaw 73], [Hanson 81], [Tennent 82], and [Clarke et al. 80] the role of block structure in Algol-60, Pascal and Ada is discussed. In these languages block structure appears in the form of nested procedures and blocks. In languages like Simula 67 ([Simula]) and Beta ([Beta]) block structure is also present in the form of nested classes.

A class describes the structure (intension) of a category of objects. In Smalltalk an object is characterized by the set of methods (procedures) that it will respond to. In Simula and Beta an object is characterized by a set of attributes, which may be variables, procedures and classes. It will be demonstrated that the use of classes as attributes of an object is useful from both a conceptual and technical viewpoint. Block structure provides locality: By declaring a procedure local to a class or procedure reflects the fact that the procedure only has meaning as part of its enclosing procedure or class. In the same way by declaring a class local to another class states that the local class is only meaningful in a limited context, i.e. as part of an object.

In section 2 block structure as used in this paper is described. The rest of the paper contains a number of examples of using block structure in the form of nested classes. The examples will be given in Beta. In Simula the use of nested classes is limited by a number of restrictions. Beta does not have these restrictions.

\section{Block Structure}

First the terminology used in the paper will be described. This includes the programming language notation used for describing the examples. Next the role of block structure will be discussed and finally we shall comment on some of the discussion of block structure in the litterature.

\section{Terminology}

In this paper block structure means textually nested procedures, classes and blocks as in Simula and Bcta. The term object will be used as a common name for instances of procedures, classes and blocks. By block is meant the Algol-60 type of statement. A block-activation is an instance of a block and covered by the term object.

The language used for describing the examples is restricted to a minimum. The following syntax is used:

<program> ::= <object-description>

$<$ class-declaration> ::= <name> : class <object-description>

$<$ procedure-declaration $>:=<$ name $>$ :proc <formal-parameters $><$ object-description $>$ 
$<$ formal-parameters $>::=$ (<input-parameters $>$ ) $\rightarrow$ (<output-parameters $>$ )

$<$ variable-declaration> $::=<$ name-list> : <object-specification>

<object-specification> $::=$ <class-name> | <object-description>

<object-description> ::= <super-class $>$ begin <declaration-list>do <imperative-list $>$ end

<super-class> ::= <class-name> | empty

$<$ imperative $::=<$ procedure-activation $>$

<object-description>

$<$ variable-name $>:=<$ expression $>$

A class-declaration and a corresponding variable-declaration may then appear as in the following program. Comments are enclosed by $\{$ and $\}$.

begin

$C$ :class $S\{\mathrm{~S}$ is the superclass of $\mathrm{C}\}$

begin \{C-objects have 3 attributes

$A 1: D ;\{\mathrm{A} 1$ is an instance of class $\mathrm{D}\}$

$P$ :proc $(X$ : integer, $Y:$ boolean $) \rightarrow(Z:$ integer $)$;

begin $\{P$ is a procedure attribute

\{with two input parameters, $X, Y$ \}

\{and one output parameter, $\mathrm{Z}$.\}

$\{\mathrm{X}, \mathrm{Y}, \mathrm{Z}$ are themselves instance of classes\}

end ;

$T:$ class $\ldots\{T$ is a class-attribute $\}$

do $I\{I$ is an imperative that may be executed $\}$

end ;

$a: C ;\{\mathrm{a}$ is an instance of class $\mathrm{C}, \mathrm{a} \mathrm{C}$-object $\}$

do ...

end

In the example language variables have a type in the form of a class name. This means that the variable will denote an instance of that class or one of its subclasses. This is the same as in Simula.

The generation time of objects has not been defined. An object may be generated together with the object containing the variable. Or objects may be generated by executing a new-imperative (like in Simula and Smalltalk). Both possibilities exist in Beta.

The example language also includes so-called singular objects, which are objects described directly without referring to a class or procedure:

begin $\{\mathrm{Bl}\}$

$V$ : begin $\{\mathrm{B} 2\} I$ : integer end ;

do $V . I:=7$;

begin $\{\mathrm{B} 3\}$

$X$ : integer

do $X:=V . I ; \ldots$

end ;

end

The whole program is a singular object described by $\mathrm{Bl}$. The variable $V$ is a singular object described by B2. B3 is a singular object describing an imperative in the form of an Algol-60 like block.

Most of the examples in this paper may (except for syntax) be expressed in the Beta programming language. Constructs not available in Beta are explicitly mentioned.

\section{The role of Block Structure}

In the object oriented programming style, a program execution is viewed as a simulation model of 
some real or imaginary system, called the referent-system ([Delta]). In order to create a model of the referent-system concepts covering the relevant phenomena must be developed.

For a concept we shall use the classic terms which are: the name used to denote the concept, the intension: the properties of the phenomena covered by the concept, and the extension: the set of phenomena covered by the concept.

The model system (or program execution) contains elements corresponding to the phenomena and concepts selected as important for the desired perspective on the referent-system. Classes and procedures model concepts and objects model phenomena.

Abstraction mechanisms in programming languages are important. Most object oriented programming languages support the three fundamental subfunctions of abstraction: classification, aggregation and generalization. The inverse functions exemplification, decomposition and specialization are similarly supported.

A class definition is a description of the intension of the instances (extension) of the class. This description includes: one or more superclasses specifying which classes/concepts that the new class specializes, a set of attributes characterizing instances of the new class, and an imperative-list that describes an action-sequence associated with instances of the class.

The attributes of a class/procedure may be described by referring to other classes/procedures, i.e. aggregation is taking place. The attributes may describe components that are a fixed part of the surrounding object, or components which are references to objects. Here block-structure or locality is important: Locality makes it possible to describe that an object is characterized by a concept in the form of a local class or procedure. This restricts the existence of instances of such local classes or procedures to the lifetime of the enclosing object in which they are defined. In the remaining sections of this paper a number of examples of this will be given.

Block structure is not a mechanism for "programming in the large" in the sense that a program should be structured as a large program consisting of nested procedures and classes. A programming language must contain facilities for modularizing a program into minor parts. Especially aggregation should be supported by a construct like the Ada package allowing another hierarchy than block structure. In [BETA 83a] a language independent mechanism for program modularization is described.

A concept/abstraction is timeless in the sense that it has no state that changes over time. Since classes are used to model concepts, classes should not have state. An object is a phenomenon which has a state that may change over time. Objects may have the same form, i.e. belong to the same class; but they have different substance. This means that they have a different location in terms of coordinates and time. Examples af objects are people, furniture, etc.

There are however phenomena which do not have substance ([Delta],[Beta]). A process (a partially ordered set of events) is an example of a phenomenon appearing in a program execution. The concepts covering such phenomena are typically modelled by procedures or concurrent process descriptions. Values and types in programming languages model concepts where the phenomena are measurable properties of objects, i.e. the substance.

\section{Discussion of Block Structure}

There are many aspects of block structure being discussed in the literature. Here we shall comment on this discussion.

- Locality. The major advantage of block structure is locality. This makes it possible to restrict the exsistence of an object and its description to the environment (object) where it has meaning.

- Scope rules. There are (at least) the following aspects of scope rules for names declared within an object:

1. They only exist when the object exist. This is a consequence of locality.

2. Access to global names and redeclaration of names. 
Global names may or may not be seen within a block. In [Wulf \& Shaw 73] it is being argued that the use of global variables within nested blocks is a source of errors.

It is considered a problem that a name can be redeclared within an internal block. There is however no reason to allow such redeclaration in case it is found to be a problem.

Also it has been critized that it may be difficult to see which global names are being used within an internal block. Again this this is not inherently tied to block structure and can be avoided. In languages like Euclid ([Lampson 77]), a block must explicitly import from the enclosing block all names being used.

As mentioned above block structure is not a mechanism intended for "programming in the large". In languages like Algol 60 and Pascal this is the case. It is merely inteded for "programming in the small". In this case the above problems tend to be minor.

3. Acces to names within a block from "outside" the block may be restricted. Hidden/protected of Simula is an example of this.

- Syntax. In [Hanson 81] it is said that:

Block structure can make even moderately large programs difficult to read. The difficulty is due to the physical separation of procedure-headings from their bodies....

In [Tennent 82] it is demonstrated that this is merely a matter of syntax. By using the syntax from Landin's ISWIM it is possible to place internal procedure declarations after the body of the block.

\section{Class Grammar}

Here we shall give an example of a class with a local class attribute. The actual example is inspired by Liskov and Zilles ([Liskov \& Zilles 74]), but is typical for a number of situations. The example defines a grammar that is going to be used for constructing a precedence parser. For this purpose a class corresponding to the symbols (tokens) of the grammar must be present and a precedence relation function must be defined. The grammar can be structured as follows:

grammar :class

$$
\begin{aligned}
& \text { begin ... \{grammar representation } \\
& \text { token :class } \\
& \text { begin ... \{token representation\} } \\
& \text { isTerminal : proc }() \rightarrow(b: \text { boolean }) ; \ldots \\
& \text { end ; } \\
& \text { isNonTerminal : proc }() \rightarrow(b: \text { boolean }) ; \ldots \\
& \text { precRel :proc }(S 1, S 2: \text { token }) \rightarrow(C: \text { char }) \\
& \text { begin ...end ; }
\end{aligned}
$$

end

It is now possible to declare instances of class grammar:

G1 : grammar; $A, B$ : Gl.token;

$G 1$ is an instance of Class grammmar and $A, B$ are instances of G1.token. For the tokens $A, B$ operations like $A$.is Terminal and $G 1 . \operatorname{precRel}(A, B)$ are possible.

Consider another set of instances:

G2 : grammar; $X, Y: G 2 . t o k e n$

$G 1$ and $G 2$ are both instances of Class Grammar. $A, B$ and $X, Y$ are not instances of the same class. $A, B$ are instances of G1.token and $X, Y$ are instances of G2.token. Intuitively this is what we want, since $A, B$ are $G 1$-tokens and $X, Y$ are $G 2$-tokens. The two class of tokens are clearly different. 
Also the two functions G1.precRel and G2.precRel are different functions.

By declaring token local to class grammar we have the possibility to distinguish between tokens from different grammars. Also since class token is local to class grammar, a token has no exsistence without a grammar. This also seems to be intuitively correct.

In Liskov and Zilles, class token is declared outside class grammar. The representation of a token is therefore not restricted to the definition of class grammar. Liskov and Ziles are aware of this problem:

Token is a good example of a type created to control access to implementation details. ....

Therefore, the new type, token, is introduced to limit the distribution of information about

how the grammar is represented.

In our opinion class token should be defined as part of class grammar since token-objects are only meaningful in relation to a specific grammar. Also knowledge about the represenation of a token should be restricted to class grammar.

\section{Functional Classes and Mutually Dependent Classes}

In this section we shall show how to suppport a Pascal or Ada like style of programming. (Called operator/operand-style in [Cox 84]).

\subsection{Functional Classes}

Simula has often been criticized for the unnatural asymmetri between operands of a function. Consider the class complex:

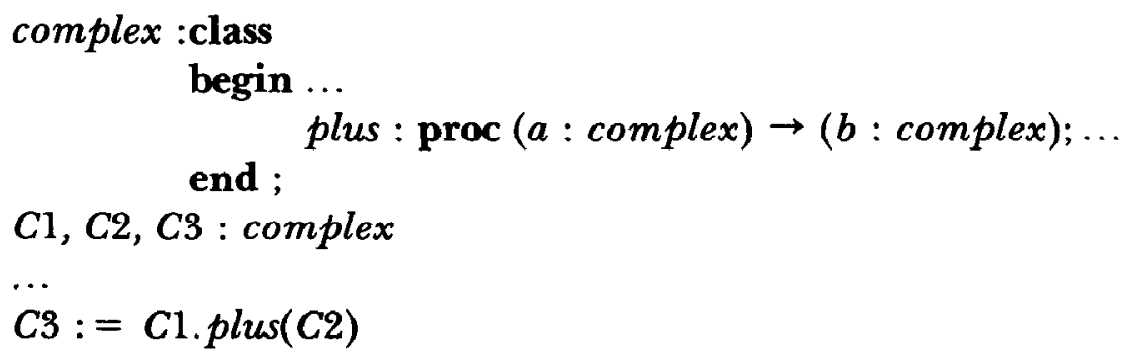

Here it seems unnatural that one of the arguments of plus serves a special purpose. This has been critized by many people. In CLU ([Liskov \& Zilles 74]) they have decided to qualify the operations by means of the class-name instead of the instance-name. The above operation would then look as follows in CLU:

$$
\text { C3 : = complex } \$ p l u s(C 1, C 2)
$$

A consequence of this is that that all operation-calls must be denoted in this way. This does not fit into the object oriented style.

In Smalltalk the assymmetri has been kept. Numbers are viewed as instances of a class and respond to messages. We are not necessarily convinced that this is a natural way of modelling numbers in a programming language. Below we shall show that the more traditional view can in fact be modelled within the Simula world. Consider the following definition of a complex package:

begin

$$
\begin{aligned}
& \text { complexPck } \text { class } \\
& \text { begin } \\
& \text { complex }: \text { class begin } I, R: \text { real end } ; \\
& \text { create }: \text { proc }(R, I: \text { real }) \rightarrow(C: \text { complex }) ; \\
& \text { begin do } C . R:=R ; C . I:=I \text { end ; } \\
& \text { plus :proc }(A, B: \text { complex }) \rightarrow(C: \text { complex; })
\end{aligned}
$$


begin do $C . I:=A . I+B . I ; C . R:=A . R+B . R$ end ;

end ;

- $C$ : complexPck;

$X, Y, Z$ : C.complex;

do

$X:=$ C.create $(1.1,2.2) ; Y:=C$. create $(3.1,0.2) ; Z:=C . p l u s(X, Y)$

end

\subsection{Mutually Dependent Classes}

It is straight forward to generalize the above technique to define mutually dependent classes, i.e. classes that must know about each others representation.

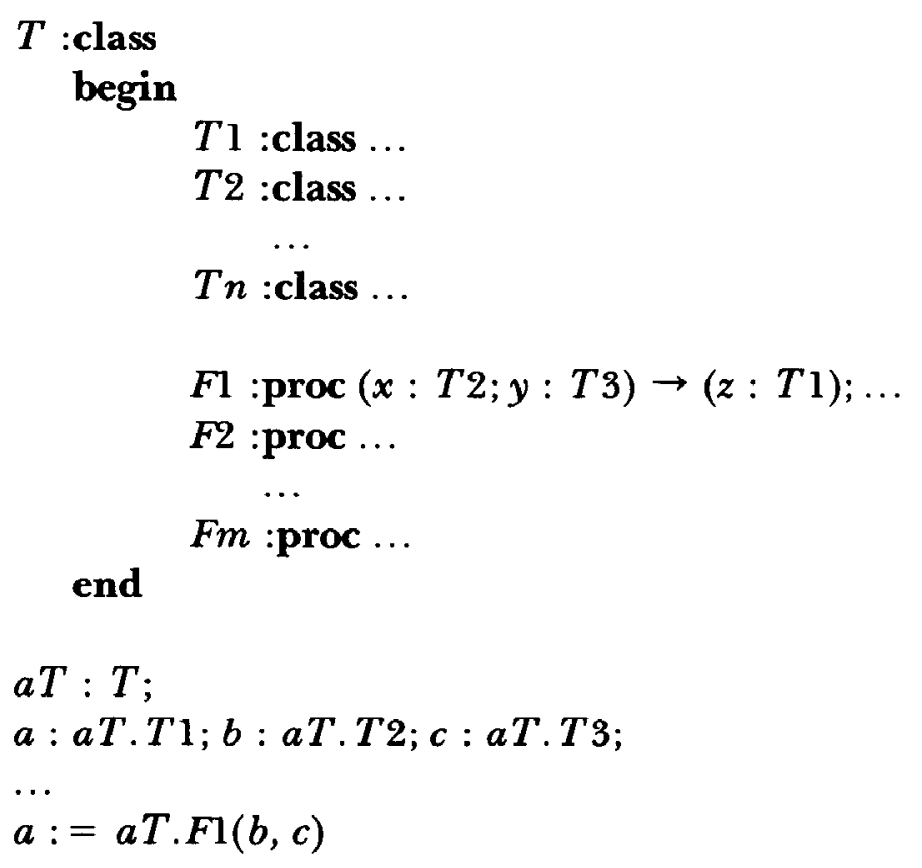

It may be awkward always to have to qualify attributes of class $T$ with $a T$, especially if there is only one instance. This may be avoided by a mechanism similar to the with-statement of Pascal or inspect-statement of Simula (the with-statement is not in Beta):

with $a T$ do

begin $a: T 1 ; \ldots$ do $a:=F 1(b, c)$ end

It may also be desirable to avoid declaring a class. This can be avoided by declaring a singular object:

$a T$ :begin

$$
\begin{aligned}
& T 1: \operatorname{class} \ldots ; T 2: \text { class } \ldots ; \\
& F 1: \text { proc } \ldots ; F 2: \text { proc } \ldots ;
\end{aligned}
$$

Since an <object-description> may contain variable declarations, a singular instance like $a T$ may contain variables. It may then be used as an Ada package. The class $T$ corresponds to an Ada generic package and the instance $a T$ to an instantiation of the generic package.

In Beta, a class (called pattern in Beta) may have virtual pattern attributes which can be used like generic formal types.

The class $T$ corresponds to an algebraic structure:

$$
T=(T 1, T 2, \ldots, T n, F 1, F 2, \ldots F m)
$$




\title{
5 The Prototype Abstraction Relation Problem
}

Consider a phenomenon that may be viewed as a prototype of a set of other phenomena. Such a prototype phenomenon may be modelled as an instance of a class describing the properties of it and other similar prototype phenomena. The prototype phenomenon has a state which is changing over time, so it seems unnatural to describe it as a subclass of the class describing its properties.

The prototype phenomenon bears a certain relation to the set of phenomena of which it is a prototype. This relation indicates that the prototype phenomenon should be modelled as a class.

The problem is known as the Prototype Abstraction Relation Problem and has been formulated by Brian Smith ([Smith 84$])$. The problem is best described by an example.

- Consider a class flightType, which defines the properties of flight descriptions in a flight tables.

- SK273 between Copenhagen and Los Angeles is an example of such a flight. SK273 is a flight that takes place every Monday, Wednesday, and Friday. The scheduled departure time is 11:30pm.

- During a period of time the properties of $S K 273$ may change. E.g. the scheduled departure time may be changed. This indicates that SK273 should be modelled as an instance of class flight Type.

- SK273 may be viewed as a a prototype of the actual flights that take place between CPH and LA. The actual flights are characterized by attributes such as actual departure time, actual flight time, etc.

- $S K 273$ may also be viewed as a class with the actual flights as instances. Also $S K 273$ could be a subclass of class flight Type.

A solution to this problem may be formulated using block structure in the form of classes as attributes.

- Class flight Type is a class describing the properties of the flight prototypes in the flight table. The attributes of instances of flight Type are source and destination of the flight, frequency, scheduled departure time, scheduled flight time, etc. In addition there is an attribute class flight describing the properties of the actual flights of the prototype. Attributes of the actual flights are departure date, actual departure time, actual flight time, departureDelay, etc.

- $S K 273$ is an instance of class flight Type.

- Instances of class SK273.flight are the actual flights between CPH and LA.

- myFlight.departureDelay will give the time that one specific actual flight has been delayed.

\section{flight Type : class}

begin source, destination : city;

frequency : set OfWeekDay;

departure Time : timeOfDay;

flight Time : timePeriod;

flight :class

\author{
begin departuredate : date; \\ actualDeparture Time : timeOfDay; \\ actualflight Time : timePeriod; \\ departureDelay :proc : timePeriod; \\ begin return actualDeparture Time- \\ departure Time
}


end ;

end ;

end ;

SK273 :flight Type

where source $=$ Copenhagen, destination $=$ LosAngeles,

frequency $=$ Mon, Wed, Fri,

departure Time $=12: 30 \mathrm{pm}$,

flight Time $=11 \mathrm{~h}: 30 \mathrm{~m}$;

myFlight :SK273.flight

where departureDate $=$ Feb.1.84,

actualDeparture Time $=12: 45 p m$

actualFlight Time $=11 \mathrm{~h}: 15 \mathrm{~m}$;

The where-construct is used to indicate bindings of the variables in an object. In Beta this can be expressed by means of virtual patterns.

\section{Smalltalk Metaclasses}

In Smalltalk-80 all system components are represented by objects. Since all objects are instances of a class, the classes themselves are represented by instances of so-called metaclasses. Metaclasses give rise to both a philosofical and technical discussion.

As stated in section 2, a class is a model of an abstraction and abstractions are timeless in the sense that they have no state changing over time.

A Smalltalk class viewed as an object may have a state changing over time.

It is true that concepts develops over time and may be changed. A concept is thus only "stable" during a period of time. When a concept changes, it is the whole structure (intension) of the concept that changes. This cannot be modelled by Smalltalk metaclasses. Here it is only possible to model changes by means of class variables. Changing the structure of a class corresponds to editing the definition of a class. This kind of change is of course possible in any programming system, but in Smalltalk-80 (and other systems) this has not been reflected in any philosofical model.

Technically metaclasses are useful for describing variables and methods (class variables and class methods) that are common to all instances of a class.

Below we shall show that the pure technical use of metaclasses can be simulated by block structure. Consider the following description of a Smalltalk class

$\begin{array}{ll}\text { class name } & T \\ \text { superclass } & S \\ \text { instance variable names } & N M \\ \text { class variable names } & X Y \\ \text { class methods } & \text { new } \\ \text { instance methods } & M 1\end{array}$

This example can be simulated by the following example:

metaT :class metas

begin

$X, Y: \ldots$

new : proc () $\rightarrow(R: T) ; \ldots$

$T$ :class $S$ 


begin


$\quad$ end ; $M 1: \ldots$
end ; $\quad$

$a M e t a T:$ metaT

The class metaT corresponds to the metaclass for class $T$. The instance aMetaT corresponds to class $T$ viewed as an object. There is however a difference between the instance aMetaT and the class $T$. $T$ is described as a class attribute of aMetaT.

In the example, the variables $X, Y, N, M$ have not been given any qualification (type) following the Smalltalk tradition.

Instances of class aMetaT.T may be created by aMetaT.new.

We shall not here postulate that this is better than metaclasses in Smalltalk. The purpose is just to show that the more technical usage of metaclasses, can be simulated with more traditional language constructs.

There is another technical use of metaclasses in the sense that all system components are represented by instances of classes. The text/description of a class is itself an object. This is useful in a programming system. Here it is however important that the levels be separated. The level of the program execution and the level of program modification are different and should be kept separate.

\section{Conclusion}

The purpose of this paper has been to show that block structure as found in Simula 67 and Beta, but abandoned in Smalltalk-80, is a natural and powerful mechanism. When modelling phenomena, it is useful to be able to characterize an object by means of a class. In any case block structure is useful for a number of technical problems in programming. This has been demonstrated by giving examples of the use of block structure.

Class grammar is perhaps the most common type of example where a class (token) is described locally to a grammar. The prototype abstraction relation problem is just a special version of this problem.

The examples in section 4 shows that even the operator/operand style and functional style of programming can be supported withing a language which is primarily intended for the object oriented style of programming. As pointed out by others, ([Cox 84],[Nygaard \& Sörgaard 85]), a programming languages should not only support one style.

Smalltalk- 80 metaclasses seem to be a technical trick which may be handled by more traditional constructs.

In [BETA 85] block structure has been used to define objects containing locally defined objects. Such compound objects are shown to be an alternative to guarded input/output commands.

In Beta the notions of class, procedure and type have been unified into one abstraction mechanism, the pattern. Instances of a pattern may then be used as objects, procedure activations or variables. A single general concept thus serves a number of purposes. In contrast Ada contains a number of concepts that are similar, but have been treated differently in many situations. This has been critizised by Peter Wegner ([Wegner 83]).

Acknowledgement. This work has evolved during discussions with Jørgen Lindskov Knudsen, Bent Bruun Kristensen, Birger Møller-Pedersen, Kristen Nygaard, Kristine Stougård Thomsen and Terry Winograd. 


\section{References}

1. [Beta 83a] B.B. Kristensen, O.L Madsen, B. Møller-Pedersen, K. Nygaard: Syntax Directed Program Modularization. In: Interactive Computing Systems (ed. P. Degano, E. Sandewall), North-Holland, 1983.

2. [Beta 83b] B.B. Kristensen, O.L. Madsen, B. Møller-Pedersen, K. Nygaard: Abstraction Mechanisms in the BETA Programming Language. Proceedings of the Tenth ACM Symposium on Principles of Programming Languages, January 24-26 1983, Austin, Texas.

3. [Beta 85] B.B. Kristensen, O.L. Madsen, B. Møller Pedersen, K. Nygaard: Multisequential Execution in the BETA Programming Language. Sigplan Notices, Vol. 20, No. 4, April 1985.

4. Clarke L.A., J.C. Wiledon, A.L. Wolf: Nesting in Ada Programs is for the Birds. Proc. ACM Symposium on the Ada Programming Language, SIGPLAN Notices, 11, 139-145 (1980).

5. Cox B.R: Message/Object, An Evolutionary Change. IEEE SOFTWARE, Jan. 1984.

6. Goldberg A., D. Robson: Smalltalk-80, The Language and its Implementation. Addison Wesley 1985.

7. [Delta] E. Holbæk-Hanssen, P. Håndlykken, K. Nygaard: System Description and the Delta Language. Norwegian Computing Center, Publ. no 523, 1975.

8. Hanson D.R.: Is Block Structure Necessary. Software Practice and Experience, Vol. $11853-866$ (1981).

9. Lampson B.W. et al: Report on the Programming Language Euclid. SIGPLAN Notices 12, 2 (1977).

10. Liskov B., S. Zilles: Programming with Abstract Data Types. Sigplan Notices, Vol. 9, No. 4, 50.59 (1974).

11. Nygaard K., P. Sørgärd: Perspective, A Key Concept in Informatics. Working Conference on Development and use of Computer-Based Systems and Tools, Aarhus, Denmark, Aug. 1985.

12. [Simula] O.-J. Dahl, B. Myrhaug, K. Nygaard: SIMULA 67 Common Base Language. Norwegian Computing Center, Oslo 1968.

13. Smith B.: Personal Communication. Stanford 1984.

14. Tennent R.D.: Two Examples of Block Structuring. Software-Practice and Experience, Vol. 12, 385-392 (1982).

15. Wegner P.: On the Unification of Data and Program Abstraction in Ada. Proceedings of the Tenth ACM Symposium on Principles of Programming Languages, January 24-26, 1983, Austin, Texas.

16. Wulf W.A., M. Shaw: Global Variables Considered Harmful. Sigplan Notices, Vol. 8, 28-34 (1973). 